\title{
Do the legs of magnetic clouds contain twisted flux-rope magnetic fields?
}

\author{
Article \\ Published Version \\ Creative Commons: Attribution 3.0 (CC-BY) \\ Open Access
}

Owens, M. (2016) Do the legs of magnetic clouds contain twisted flux-rope magnetic fields? The Astrophysical Journal, 818 (2). 197. ISSN 0004-637X doi:

https://doi.org/10.3847/0004-637X/818/2/197 Available at https://centaur.reading.ac.uk/56699/

It is advisable to refer to the publisher's version if you intend to cite from the work. See Guidance on citing.

To link to this article DOI: http://dx.doi.org/10.3847/0004-637X/818/2/197

Publisher: American Astronomical Society

All outputs in CentAUR are protected by Intellectual Property Rights law, including copyright law. Copyright and IPR is retained by the creators or other copyright holders. Terms and conditions for use of this material are defined in the End User Agreement.

\section{www.reading.ac.uk/centaur}

\section{CentAUR}

Central Archive at the University of Reading

Reading's research outputs online 


\title{
DO THE LEGS OF MAGNETIC CLOUDS CONTAIN TWISTED FLUX-ROPE MAGNETIC FIELDS?
}

\author{
M. J. OWENS \\ Space and Atmospheric Electricity Group, Department of Meteorology, University of Reading, Earley Gate, P.O. Box 243, Reading RG6 6BB, UK \\ Received 2015 December 26; accepted 2016 January 18; published 2016 February 18
}

\begin{abstract}
Magnetic clouds (MCs) are a subset of interplanetary coronal mass ejections (ICMEs) characterized primarily by a smooth rotation in the magnetic field direction indicative of the presence of a magnetic flux rope. Energetic particle signatures suggest MC flux ropes remain magnetically connected to the Sun at both ends, leading to widely used model of global MC structure as an extended flux rope, with a loop-like axis stretching out from the Sun into the heliosphere and back to the Sun. The time of flight of energetic particles, however, suggests shorter magnetic field line lengths than such a continuous twisted flux rope would produce. In this study, two simple models are compared with observed flux rope axis orientations of $196 \mathrm{MCs}$ to show that the flux rope structure is confined to the MC leading edge. The MC "legs," which magnetically connect the flux rope to the Sun, are not recognizable as MCs and thus are unlikely to contain twisted flux rope fields. Spacecraft encounters with these non-flux rope legs may provide an explanation for the frequent observation of non-MC ICMEs.
\end{abstract}

Key words: solar-terrestrial relations - solar wind - Sun: activity - Sun: coronal mass ejections (CMEs) Sun: heliosphere - Sun: magnetic fields

\section{INTRODUCTION}

Magnetic clouds (MCs) are a subset of interplanetary coronal mass ejections (ICMEs) primarily characterized by a smooth rotation of the magnetic field direction (Burlaga et al. 1981), which is interpreted as a magnetic flux rope convecting past the spacecraft (Burlaga 1988; Lepping et al. 1990). MCs have received a great deal of attention for two reasons. First, they are the drivers of the most severe space weather (Gonzalez et al. 1999 and references therein), primarily due to the large out-of-ecliptic magnetic field that the flux rope can produce. Second, the flux rope structure means the global properties of MCs can be inferred from single-point in situ observations, allowing ready comparison with the solar source of the eruption (e.g., Bothmer \& Rust 1997).

The frequent observation of counter-streaming suprathermal electrons (Feldman et al. 1975) within MCs suggests that both ends of the flux rope remain connected to the Sun as the structure propagates out through the heliosphere (Gosling et al. 1987). This has led to a widely used model of the largescale structure of a MC as an axial field extending out from the Sun, to the in situ observer and back, with highly twisted toroidal flux rope field along its length. Examples are shown in Figure 1. Of course, the "legs" of the MC which connect the leading edge to the Sun will rotate with the Sun and thus approximately follow the Parker spiral (Parker 1958). This has led to a number of modifications to cylindrical flux rope models to account for axial curvature (Marubashi \& Lepping 2007; Démoulin \& Dasso 2009; Romashets \& Vandas 2009; Owens et al. 2012).

Assuming the flux rope structure extends throughout the legs of the MC, the magnetic field-line length connecting the Sun and observer will be much longer near the outer edge of flux ropes than at the flux rope axis. This means suprathermal electrons near the outer edge of the flux rope will have much longer travel times from Sun to observer and thus greater opportunity for the field-aligned beam, or "strahl," to undergo pitch-angle scattering (Owens et al. 2008). While there is some statistical evidence of broader strahls at the flux rope edge than axis, the effect is much weaker than predicted using the legged flux rope model for MCs (Owens et al. 2009). A similar discrepancy is found in rare events where solar energetic particles (SEPs) are observed within the flux rope structure of MCs. The observed time-of-flight dispersion of the SEPs suggests the path length of particles between the Sun and $1 \mathrm{AU}$ is shorter than the tightly wound helical field expected from a flux rope stretching the complete distance from the Sun to observer (Farrugia et al. 1993; Larson et al. 1997; Chollet et al. 2007; Kahler et al. 2011a, 2011b; Dresing et al. 2015).

In this study, the local axis orientations from a number of large surveys of MCs are also shown to be inconsistent with the "global" flux rope picture described above. The implications of this result for the large-scale structure of MCs are discussed.

\section{MODELS OF GLOBAL MC STRUCTURE}

In this study two simple models for the large-scale structure of MCs are compared in a purely statistical sense. Note that the aim here is not to produce a model which provides an accurate fit to individual MC encounters, such as with the techniques of Démoulin \& Dasso (2009), Hidalgo et al. (2002), Hu \& Sonnerup (2001), Owens et al. (2006), etc.

The first model is the global flux rope shown in Figure 2(a). The axis of the MC is assumed to consist of an approximately circular front, as often seen in coronagraph (Hundhausen 1993) and heliospheric imager (Savani et al. 2010) observations, with Parker spiral-aligned legs connecting the circular front to the Sun at either end. As suggested by the cartoons of Figure 1, the flux rope structure extends along the entire length of the axis, back to the coronal source surface. The second model is the "leading edge" flux rope shown in Figure 2(b). The axial magnetic field follows the exact same shape as the first model, but the flux rope structure is assumed to be limited to the circular front, with the legs consisting of untwisted magnetic flux, approximately orientated with the Parker spiral. In both cases, the MC axis is assumed to lie close to the ecliptic plane.

Synthetic spacecraft encounters with the model MCs are generated at 1000 equally spaced locations in heliographic longitude relative to the flux rope position. For simplicity, the spacecraft encounter is approximated as a radial cut through the 

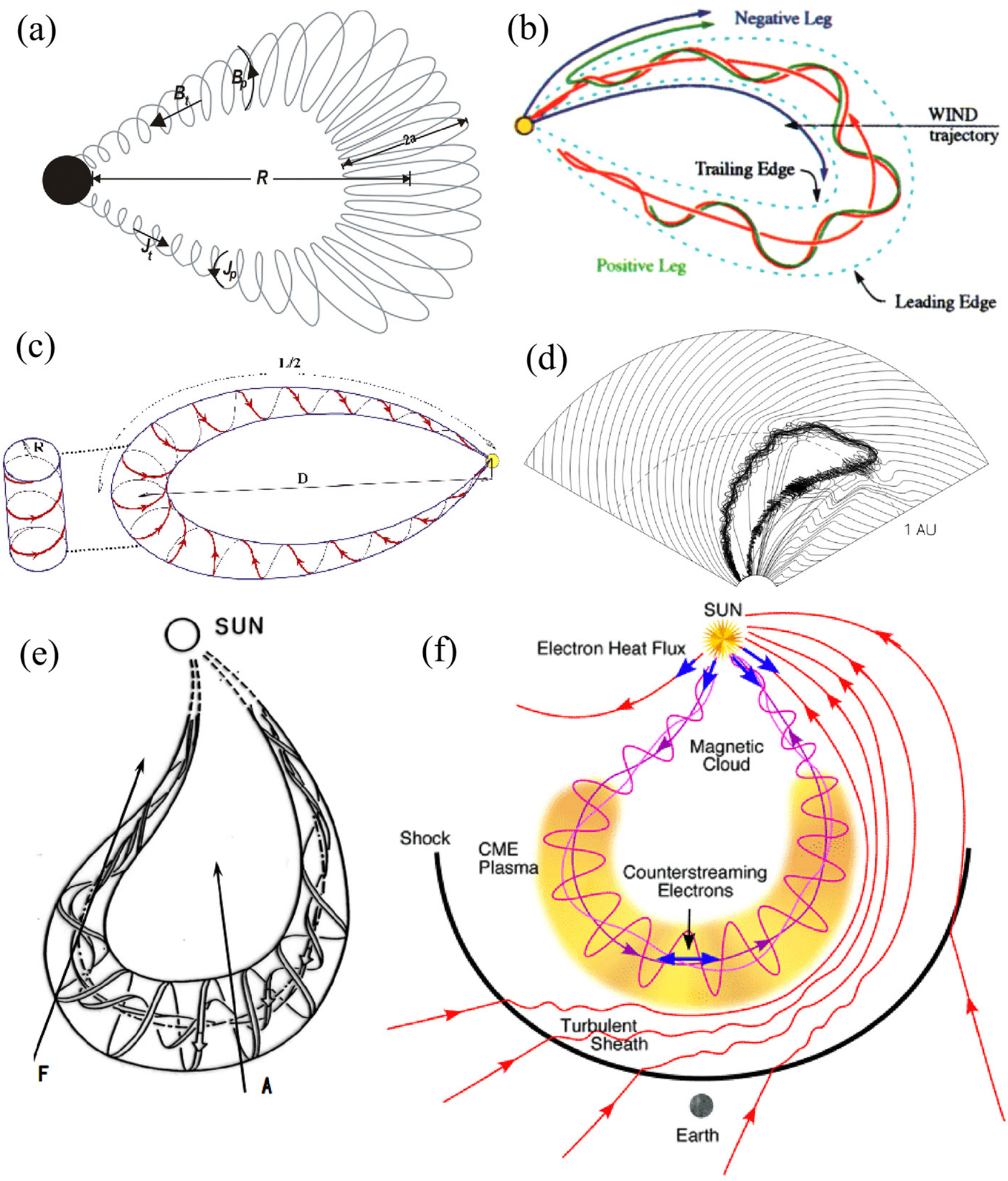

Figure 1. Cartoons and models of the global structure of magnetic clouds. (a) The near-Sun structure of a coronal mass ejection, from Subramanian et al. (2014). (b) A partially "open" magnetic cloud inferred from solar energetic particle observations, from Larson et al. (1997). (c) A model for fitting in situ magnetic cloud observations, from Démoulin \& Dasso (2009). (d) A numerical simulation of a flux rope in interplanetary space, from Vandas et al. (2002). (e) and (f) Sketches of the global structure of magnetic clouds from Marubashi \& Lepping (2007) and Zurbuchen \& Richardson (2006), respectively. 

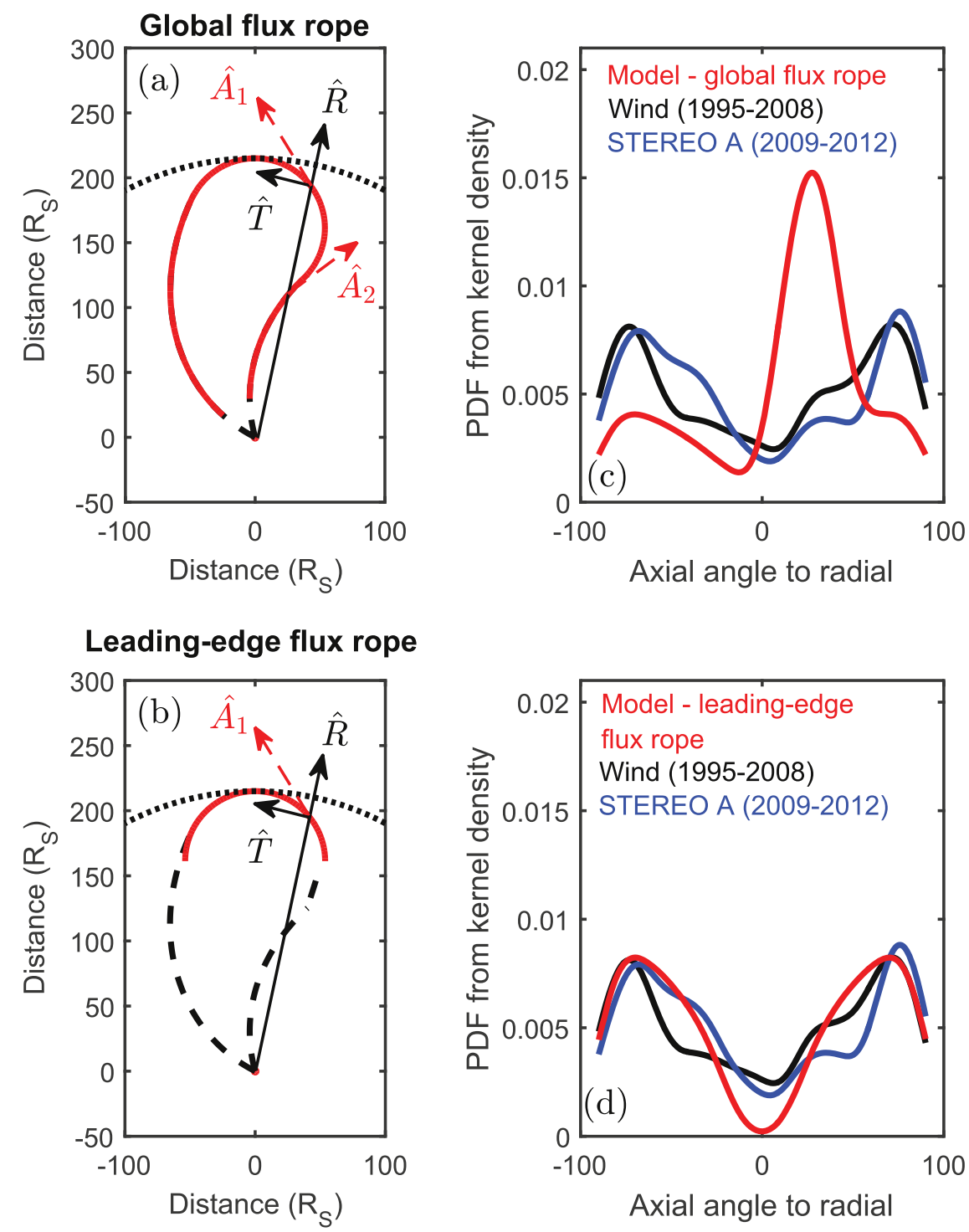

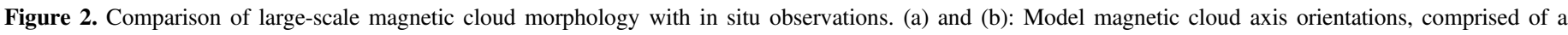
circular front and Parker-spiral "legs." Red lines show the extent of the flux rope structure, the black dashed line shows untwisted flux. The black dotted arc shows $1 \mathrm{AU}$. (c) and (d): Occurrence frequency of flux rope axis orientations from the synthetic spacecraft encounters with the model (red) and from Wind (black) and STEREO (blue) observations. Spacecraft observations agree much more closely with the leading-edge flux rope model.

model, rather than a time evolution past a fixed point in space. An example is shown in Figure 2(a). As the model only accounts for axis orientation and not magnetic field polarity, there is a $180^{\circ}$ ambiguity in the direction of the axial magnetic field and thus the axis orientation is taken to be in the positive $R$ direction in heliocentric $R T N$ coordinates. The spacecraft location shown in Figure 2(a) produces two flux rope encounters within the leg encountered by the spacecraft, one positive and one negative $T$ components of the axis orientation. The same location in Figure 2(b) produces a single flux rope encounter with an axis orientation in the positive $T$ direction. For each flux rope encounter, the angle of the axis to the $R$ direction (through positive $T$ ) is recorded and the resulting probability distribution functions (PDFs) from kernel-density estimates are shown as red lines in Figures 2(c) and (d). There is a clear difference in the expected occurrence of flux rope axis orientations from the two models, with the global flux rope model showing a highly asymmetric distribution of axis orientations about the $R$ direction, with strong peak close to the Parker spiral direction at approximately $-40^{\circ}$. The distribution of axes about the $R$ direction is symmetric for the leading-edge flux rope model, peaking around $+/-70^{\circ}$ and forming a minimum at $0^{\circ}$.

\section{OBSERVATIONS OF MCS}

Two existing catalogs of MC observations are used. The Lepping catalog (Lepping et al. 2006) is available from http:// lepmfi.gsfc.nasa.gov/mfi/mag_cloud_pub1.html. It lists 121 MCs observed by the Wind spacecraft between 1995 and 2008, with axis orientations obtained by fitting the observed magnetic field vectors with a force-free magnetic flux rope model. (Note axis orientations are in Geocentric Solar Ecliptic coordinates, which have been converted to RTN for this study.) The Jian catalog (Jian et al. 2013) is available from http://www-ssc. igpp.ucla.edu/forms/stereo/stereo_level_3.html. It lists 75 MCs observed by the STEREO A spacecraft between 2009 


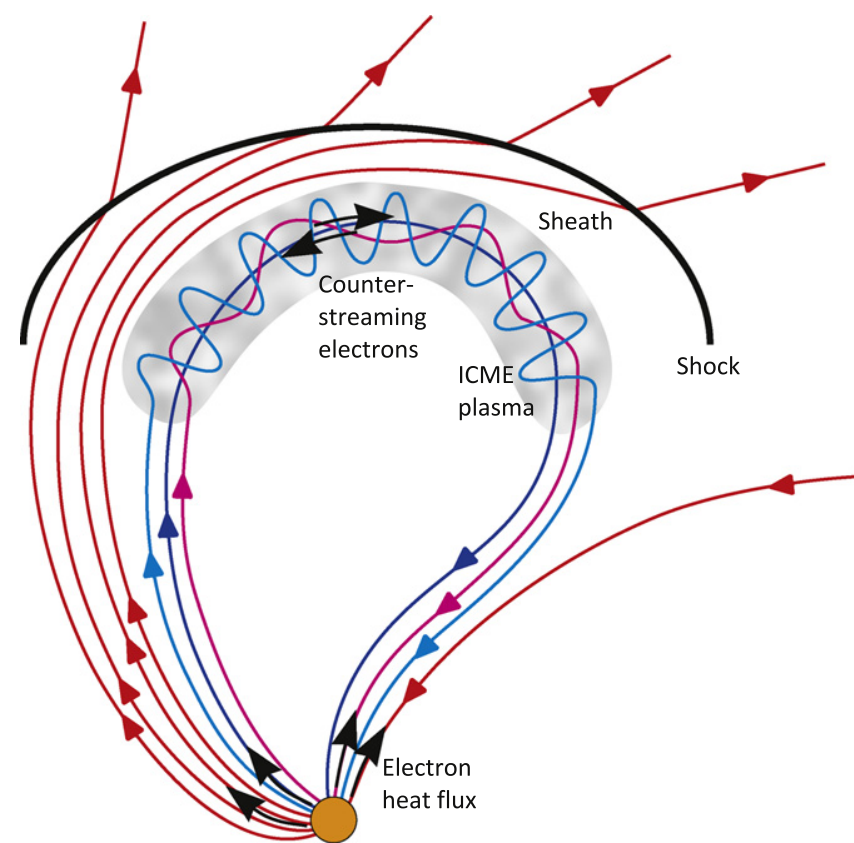

Figure 3. A modified version of the Zurbuchen \& Richardson (2006) cartoon of global magnetic cloud structure, in which the twisted flux rope structure is confined to the leading edge of the event. Blue/purple lines show magnetic flux associated with the magnetic cloud, whereas red lines show the ambient heliospheric magnetic field. Black arrows indicate the direction of the suprathermal electron strahl.

and 2012. Both of these catalogs were recently reproduced as supplementary material in the study of Ruffenach et al. (2015).

To enable comparison with the model output, the axis orientation for each MC is expressed as the angle in the $R T$ plane to the $R$ direction. The PDFs from kernel density estimates are shown as the black (Lepping) and blue (Jian) curves in Figures 2(c) and (d). Both catalogs show approximately symmetric distributions about the $R$ direction, with peaks around $+/-70^{\circ}$ and minima at $0^{\circ}$.

\section{DISCUSSION AND CONCLUSIONS}

This study considered two simple models for the global structure of MCs. The first is the commonly used global model, in which a magnetic flux rope extends completely through interplanetary space, from the Sun to observer (e.g., at 1 AU) and back to the Sun. The second is a leading-edge flux rope, in which the magnetic flux rope is only present at the leading edge of the eruption and untwisted magnetic flux connects it to the Sun at either end. The expected distributions of flux rope axes from these two models were compared with spacecraft observations of $200 \mathrm{MCs}$. The leading-edge model shows far better agreement, in particular with the approximately symmetric distribution of flux rope axes about the radial direction, with peaks close to the $+/-T$ direction in $R T N$ heliocentric coordinates. On this basis, a minor modification to the comprehensive Zurbuchen \& Richardson (2006) cartoon of large-scale MC structure is suggested in Figure 3.

The flux-rope magnetic field within magnetic fields can be formed by reconnection with rising, sheared coronal magnetic loops (Gosling 1990). Assuming this reconnection is limited to the coronal mass ejection launch, the legs of MCs, which are associated with material leaving the Sun days after the CME launch, would not be expected to contain twisted magnetic flux.
The lack of twisted flux rope along the MC legs means that previous estimates of the toroidal flux carried by solar ejecta (e.g., Lynch et al. 2005) may have been overestimated by around a factor 3 to 5 , though this may be approximately offset by an equivalent underestimate in the cross-sectional extent of MCs (Riley \& Crooker 2004; Owens 2008). This could have implications for the association between MCs and their source regions on the Sun (e.g., Qiu et al. 2007). Estimates of the axial flux content of MCs, which is central to the heliospheric flux budget (Low 2001; Owens \& Crooker 2006), are unaffected by the magnetic field structure in MC legs.

The lack of flux rope structure in MC legs could explain the paucity of "double flux rope" encounters, such as that shown in Figure 2(a). The best examples of this phenomenon tend to occur further out in the heliosphere (Rees \& Forsyth 2004), where dynamical effects such as stream interaction are likely to have a larger effect on the global structure of MCs. At 1 AU, the leading-edge model means only a single encounter with a flux rope is possible in the majority of events. A double encounter with the same flux rope may become even less likely when the axis has a large out-of-ecliptic component.

The existence of "legs" of untwisted magnetic flux may provide a ready explanation for the large number of non-flux rope ICMEs which are observed by in situ spacecraft (Riley \& Richardson 2013). These non-flux rope legs may still have high magnetic field intensity, as they must contain the same amount of flux as the twisted rope which exists at the leading edge of the MC. They may also exhibit reduced proton temperature due to increased expansion relative to the ambient solar wind as a result of high magnetic pressure. It is unclear whether they should contain any anomalous composition or charge-state signatures (Lepri \& Zurbuchen 2004). These speculations, however, need to be observationally tested. An analysis of multi-spacecraft observations, particularly STEREO A, ACE and STEREO B during the early phase of the STEREO mission, is currently underway and will be reported as part of a future study.

M.O. is part-funded by Science and Technology Facilities Council (STFC) grant number ST/M000885/1 and acknowledges support from the Leverhulme Trust through a Philip Leverhulme Prize.

\section{REFERENCES}

Bothmer, V., \& Rust, D. M. 1997, in AGU Monogr. 99, Coronal Mass Ejections, ed. N. Crooker, J. A. Joselyn, \& J. Feynman (Washington, DC: AGU), 139

Burlaga, L. F. 1988, JGR, 93, 7217

Burlaga, L. F., Sittler, E., Mariani, F., \& Schwenn, R. 1981, JGR, 86, 6673 Chollet, E. E., Giacalone, J., Mazur, J. E., \& Al Dayeh, M. 2007, ApJ, 669, 615 Démoulin, P., \& Dasso, S. 2009, A\&A, 498, 551

Dresing, N., Gomez-Herrero, R., Heber, B., et al. 2015, A\&A, 586, A55

Farrugia, C., Richardson, I., Burlaga, L., Lepping, R., \& Osherovich, V. 1993, JGR, 98, 15497

Feldman, W. C., Asbridge, J. R., Bame, S. J., Montgomery, M. D., \& Gary, S. P. 1975, JGR, 80, 4181

Gonzalez, W. D., Tsurutani, B. T., \& Clúa de Gonzalez, A. L. 1999, SSRv, 88,529

Gosling, J. T. 1990, in Physics of Magnetic Flux Ropes, ed. C. T. Russel, E. R. Priest, \& L. C. Lee (Washington, DC: AGU), 343

Gosling, J. T., Baker, D. N., Bame, S. J., Feldman, W. C., \& Zwickl, R. D. 1987, JGR, 92, 8519

Hidalgo, M. A., Cid, C., Vinas, A. F., \& Sequeiros, J. 2002, JGR, 107, 2156 $\mathrm{Hu}$, Q., \& Sonnerup, B. U. O. 2001, GeoRL, 28, 467

Hundhausen, A. J. 1993, JGR, 98, 13177 
Jian, L., Russell, C., Luhmann, J., Galvin, A., \& Simunac, K. 2013, in AIP Conf. Proc. 1539, Solar Wind Observations at STEREO: 2007-2011 (Melville, NY: AIP), 191

Kahler, S., Krucker, S., \& Szabo, A. 2011a, JGR, 116, A01104

Kahler, S. W., Haggerty, D. K., \& Richardson, I. G. 2011b, ApJ, 736, 106

Larson, D. E., Lin, R. P., McTiernan, J. M., et al. 1997, GeoRL, 24, 1911

Lepping, R., Berdichevsky, D. B., Wu, C.-C., et al. 2006, AnGeo, 24, 215

Lepping, R. P., Jones, J. A., \& Burlaga, L. F. 1990, JGR, 95, 11957

Lepri, S. T., \& Zurbuchen, T. H. 2004, JGR, 109, A01112

Low, B. C. 2001, JGR, 106, 25141

Lynch, B. J., Gruesbeck, J. R., Zurbuchen, T. H., \& Antiochos, S. K. 2005, JGR, 110, A08107

Marubashi, K., \& Lepping, R. P. 2007, AnGeo, 25, 2453

Owens, M., Démoulin, P., Savani, N., Lavraud, B., \& Ruffenach, A. 2012, SoPh, 278, 435

Owens, M. J. 2008, JGR, 113, A12102

Owens, M. J., \& Crooker, N. U. 2006, JGR, 111, A10104
Owens, M. J., Crooker, N. U., \& Horbury, T. S. 2009, AnGeo, 27, 4057

Owens, M. J., Crooker, N. U., \& Schwadron, N. A. 2008, JGR, 113, A11104

Owens, M. J., Merkin, V. G., \& Riley, P. 2006, JGR, 111, A03104

Parker, E. N. 1958, ApJ, 128, 664

Qiu, J., Hu, Q., Howard, T. A., \& Yurchyshyn, V. B. 2007, ApJ, 659, 758

Rees, A., \& Forsyth, R. J. 2004, GeoRL, 31, 6804

Riley, P., \& Crooker, N. U. 2004, ApJ, 600, 1035

Riley, P., \& Richardson, I. 2013, SoPh, 284, 217

Romashets, E., \& Vandas, M. 2009, A\&A, 499, 17

Ruffenach, A., Lavrand, B., Farrugia, C. J., et al. 2015, JGR, 120, 43

Savani, N. P., Owens, M. J., Rouillard, A. P., Forsyth, R. J., \& Davies, J. A. 2010, ApJL, 714, L128

Subramanian, P., Arunbabu, K., Vourlidas, A., \& Mauriya, A. 2014, ApJ, 790,125

Vandas, M., Odstrčil, D., \& Watari, S. 2002, JGR, 107, 1236

Zurbuchen, T. H., \& Richardson, I. G. 2006, SSRv, 123, 31 